\title{
La reforma de las Naciones Unidas. La previsión y la prevención como objetivos principales
}

\author{
Alexandra Novosseloff
}

Para abordar el actual tema de la reforma de las Naciones Unidas debemos analizar con honestidad los problemas existentes. Ante todo es necesario tener en cuenta los profundos cambios ocurridos desde el fin de la Guerra Fría y ser conscientes de las limitaciones de una organización internacional y de la estructura del sistema de las Naciones Unidas. Sólo a partir de ese análisis pueden hacerse propuestas para racionalizarlo y hacer más eficiente su funcionamiento. No sólo hay que racionalizar la organización, sino abordarla desde un punto de vista que le dé propósito y significado. El artículo propone fijar como sus metas principales la previsión y la prevención, esto es, convertirla en la guardia avanzada y la conciencia del mundo. En este contexto, las Naciones Unidas son un mecanismo indispensable y valiosísimo en las relaciones internacionales.

Los enormes cambios que han tenido lugar en las relaciones internacionales desde mediados de los años 80 han situado la reforma de las Naciones Unidas en el centro de la agenda internacional. ¿Qué debe hacer para adaptarse al nuevo contexto internacional caracterizado por la globalización y la fragmentación? Da la impresión de que la Organización no se ha anticipado, ni siquiera ha acompañado, en su estructura y métodos de trabajo, a la evolución del sistema internacional. Parece haberse conformado con mantener e implementar la agenda que imperaba durante la Guerra Fría. El empleo masivo y a veces excesivo de las costosas operaciones de mantenimiento de la paz sin una estrategia política ni medios apropiados ha intensificado las críticas al sistema, acusado de dar la misma respuesta a problemas cuya naturaleza y orígenes han cambiado.

El cincuentenario de la Organización constituyó una oportunidad ideal para, en palabras del antiguo Secretario General Boutros-Ghali, efectuar la transición de "la vieja a la nueva ONU'. Sin embargo, los Estados miembro dejaron escapar esta oportunidad de discutir de manera seria y decidida el tema de la reforma y establecer una agenda detallada para su implementación ${ }^{1}$.

'La Asamblea General ha creado nada menos que un cornité y cinco grupos de trabajo para estudiar el futuro del sistema de Naciones Unidas y sus acciones: Comité Especial de la Carta de las Nacioncs Unidas y del fortalecimiento de! papel de la Organizaciön, Grupo de Trabajo de aito nivel y composición abierta sobre el 
Pero, paradójicamente, fue después del cincuentenario de la ONU cuando entre los miembros surgió un nuevo interés por la reforma. En 1996, Estados Unidos tomó la iniciativa², apoyado por el G7/G8, seguida de un calendario detallado para la implementación de las reformas del entonces recién elegido Secretario General Kofi Annan³.

Hoy día, la "Cumbre del Milenio" (celebrada entre el 6 y el 11 de septiembre de 2000) y la "Asamblea del Milenio" representan una segunda oportunidad para que los estados miembros reflexionen acerca del porvenir del sistema de las Naciones Unidas. No sólo está en juego el futuro de la ONU, sino también su propia existencia. La intención de estas reuniones era replantear, a un alto nivel, el futuro de la ONU y su adaptación al mundo que le rodea y a sus nuevas misiones. El objetivo principal de estos eventos era concienciarse de esa necesidad, además de identificar nuevos retos y las reformas que éstos suponen. En otras palabras, ¿ cómo puede la Organización adaptarse a la evolución del sistema internacional para "proporcionar al siglo XXI una ONU equipada, financiada y estructurada que sirva de forma eficaz a las personas para las que fue creada" "? Estas preguntas abordan tanto los preparativos para el siglo XXI, a fin de "controlar mejor lo impredecible", como los principios, objetivos y el futuro del sistema de Naciones Unidas en general. No se trata únicamente de una adaptación y gestión día a día. Es más bien una cuestión de previsión para tratar de establecer un sistema realmente acorde con los retos del mañana ${ }^{6}$.

fortaiccimiento del sistema de las Naciones Unidas, Grupo de Trabajo de composición abierta sobre la cucstión de la representación equitativa en el Consejo de Seguridad y el aumento del número de sus micmbros, Grupo de Trabajo de composición abierta sobre un programa de paz, Grupo de Trabajo especial de composición abicrta sobre un programa de desarrollo, Grupo de Trabajo de alto nivel y composición abierta encargado de examinar Ia situación financiera de las naciones Unidas. También se han llevado a cabo estudios externos. Entre otros: el informe Ramphal/Carisson (Our Global Neighborhood), cl informe Qureshi/Weizsäcker (The United Nations in its Second-Half-Century: A Report of the Independent Working Group on the Future of the United Nations), el informe Ogata/Volker (Financing an Effective United Nations: A Report of the Independent Working Advisory Group on UN Financing), y el informe South Centre (For a strong and democratic United Nations: A South Perspective on UN Reform), y ediciones especiales de Futures (vol. 27, $\mathrm{N}^{\mathrm{D}} 2$, marzo de 1995) sobre "The United Nations at Fifty: Policy and Financing Alternatives", editado por Harlan Cleveland, Hazcl Henderson e Inge Kaul.

${ }^{2}$ En febrero y abril los Estados Unidos publicaron dos documentos de propuestas detalladas sobre la reforma de las Naciones Unidas: US Views on Reform Meastures Necessary for Strengthening the United Nations System, (febrero de 1996), y Preparing the United Nations for its Second Fify Years (abril de 1996).

${ }^{3}$ Documentos 'Track I' (A/51/829) del 17 de marzo de 1997, y 'Track II' (A/51/950) del 14 de julio de 1997.

${ }^{4}$ Declaración del Cincuentenario de la ONU, 24 de octubre de 1995.

${ }^{s}$ Citando a Jacques Lesoume (Les mille sentiers de l'avenir, Paris, 1981).

'Entrevista con el Sr. Jérôme Bindé, Director de la Oficina de Análisis y Previsión de la UNESCO cn Paris. 


\section{Una prioridad: prever para adaptar}

Una reforma efectiva de las Naciones Unidas requiere un análisis en profundidad de su contexto internacional. Es inevitable una reflexión anticipada y esfuerzo: por tanto, 'replantearse' el período de post Guerra Fría es la principal prioridad. De hecho, no se ha organizado ninguna conferencia política internacional para tratar los cambios que han tenido lugar entre 1989 y 1992, tal y como se hizo después de la Primera y Segunda Guerra Mundial. Todo ello a pesar de los profundos cambios generados por la caída del muro de Berlín y la 'Cortina de hierro', el desmembramiento del imperio soviético y la aceleración de la globalización, que en su conjunto han producido transformaciones tan grandes como aquellas originadas por las dos guerras mundiales. Estos cambios incluyen:

\section{- $\quad$ Procesos de globalización y fragmentación7;}

- La creciente disparidad entre ricos y pobres (tanto entre estados como dentro de las sociedades) ${ }^{8}$;

- El aumento de segmentos religiosos, étnicos y culturales;

Cambios relacionados con la naturaleza de los conflictos y la ampliación de los conceptos de seguridad y desarrollo";

- La debilitación de la idea de soberanía estatal, la crisis del estado nación;

El predominio de la lógica de la fuerza; y

La revolución tecnológica y de la comunicación a 'tiempo real'.

Estos son fenómenos clave que deben formar parte del planteamiento y decisiones de la ONU, porque, como lo pone el actual Secretario General: "quedarse quieto mientras el mundo avanza es como deslizarse inútilmente hacia atrás"10.

Estos cambios han llevado a una absoluta metamorfosis de todo el sistema internacional, que es cada vez menos interestatal y cada vez más transnacional. Su centro de gravedad se ha trasladado poco a poco de Europa a Asia Pacífico. Parece que el poder económico y financiero y la capacidad de influir han sustituido al poder político y de

\footnotetext{
${ }^{7}$ Scgún Boutros Boutros-Ghali, no hay sólo un proceso de globalización, sino muchas formas de globalización y cada una tiene su propia particularidad y ritmo. V́case "21st Century Talks", UNESCO, Le Monde, 28 de abril de 1998.

Véase PNUD, Informe sobre Desarrollo Humano 1996 y 1997.

${ }^{9}$ Véanse los capítulos 2 y 3 del Informe sobre Desarrollo Humano 1994, PNUD.

${ }^{10}$ Kofi Annan, "Peace Operations and the United Nations: Preparing for the Next Century", febrero de 1996.
} 
mando. El sistema internacional ya no está regulado por el equilibrio de poder porque la influencia y el poder norteamericanos no tienen contrapeso. Según algunos observadores, los Estados Unidos ejercen por lo tanto un 'multilateralismo autoritario'" o un 'nuevo unilateralismo' 12 que les permite gestionar los asuntos mundiales según sus intereses, agenda y objetivos propios, y basar su actividad dentro del sistema internacional en un concepto instrumental de la ONU. Esta política provoca resentimiento en muchos actores que rechazan esta dominación.

Dado el rápido aumento de la interdependencia y de los medios de comunicación, los problemas mundiales hoy día son algo compartido que no se puede reducir a una causa única o a una percepción monolítica. Estos problemas no necesariamente requieren una solución global; lo importante es encontrar, como dijo Zaki Laïdi "un nivel adecuado de decisión y actuación"13.

Finalmente, el sistema internacional de post Guerra Fría se caracteriza por la desaparición de la 'amenaza' y de la lógica de la adversidad, y por un 'sistema de referencias vacío' 14 , la pérdida de sentido's ; es un sistema donde las 'pequeñas ideologías' (el individualismo, el narcisismo, la preocupación por uno mismo) han reemplazado a las 'grandes ideologías' que arrastran consigo un proyecto, esperanzas y alternativas ${ }^{16}$. Se podría decir que, a escala mundial, existe una pérdida de sentido y una falta de proyecto federativo; y que en su lugar, existen numerosos 'micro sentidos' que inducen a "la propagación de un mosaico de 'códigos' y 'reglas' (ni aceptados ni unificadores) que no respetará nadie". Esta tendencia es resultado de la fragmentación del mundo, que conduce a una "creciente atomización de la sociedad" y que propicia la "dinámica individual en lugar de las situaciones colecti-

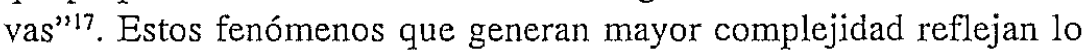
que muchos autores han denominado una 'crisis de civilización'. Esta crisis tiene tres dimensiones: la crisis del Estado nación, la crisis de la

\footnotetext{
"Irnerio Seminatore, "Les relations intemationales de l'après-guerre froide: une mutation globale", Etudes internationales, 27(3):605, septicmbre de 1996.

${ }^{12} \mathrm{G}$. Achcar (Le Monde diplomatique, octubre de 1995, p. 9) citando un articulo publicado en el International Herald Tribune, "Going It Alone and Multilateralism Aren't Leadership", 4-5 de febrero de 1995.

${ }^{13}$ Zaki Laìdi, "Le rite médiatique du G7", Libération, 15 de junio de 1996.

${ }^{14}$ Innerio Seminatorc, op. cir., p. 611.

"Véase Zaki Lä̈di, Un monde privé de sens, 1994, Paris, Fayard.

${ }^{16}$ ¡Aunque estas alternativas hayan conducido a regímenes autoritarios y a desastres humanos y humanitarios! Tambićn hay que tener en cuenta que el "decline de las grandes narrativas" cn realidad precedió al fin de la Guerra Fría, y fuc anunciado, por ejemplo, en 1979 en el ensayo profético del filósofo Jean-François Lyotard, $L a$ condition post-modeme, (Paris, Éditions de Minuit, 1979).

${ }^{17}$ Zaki Laïdi, "L'urgence est mauvaisc conseillère du prince", Libération, 11 de octubre de 1996.
} 
sociedad (que también es la crisis de la comunicación y de la comprensión) y la crisis del ser humano ${ }^{18}$.

La ONU por tanto se enfrenta hoy día a una serie de problemas que no habían previsto los fundadores de la Carta. Como dice Richard J. Poncio, "las palabras 'población', 'migración', 'hambruna', 'pobreza', y 'medio ambiente' no aparecen en la Carta de $1945^{19}$ ", y tampoco la palabra 'desarrollo', aunque en la Carta ya se menciona la necesidad de 'promover el progreso social'20 y se crea un Consejo Económico y Social. Las preocupaciones y los problemas han cambiado de forma natural desde 1945 y la ONU debe acompañar a estos cambios en las tres áreas que trata la Carta (política, económica y social). Como dijo Ghassan Salamé "a partir de ahora, los diplomáticos, investigadores y estrategas deben analizar una larga lista de situaciones concretas donde la cuestión no es encontrar la 'mano de Moscú' o a los 'agentes de la CIA' sino comprender el decline de las sociedades, la desintegración de los territorios y el fracaso de los estados" 21 . El desafío para hoy y para el futuro es "captar la faceta multidimensional de las realidades" comprensión de las situaciones complejas" "23. Para alcanzarlo, se debe tratar de no dividir las soluciones que se dan a problemas específicos y no restringirlas a una sola área. Es más, un problema no se puede abordar sólo a nivel político ya que las esferas política, social y económica están estrechamente interrelacionadas. Al contrario, "para comprender el fenómeno, deben cuestionarse las causas además de la interconexión entre los distintos actores que son la política, la guerra, la ley, la economía, la cultura, la moral... y abordar el todo con un único y sencillo enfoque"24. Esto es particularmente útil a la hora de comprender mejor las raíces y la naturaleza intraestatal de los conflictos actuales.

La Carta de 1945 no previó el problema de los conflictos intraestatales o infraestatales. Durante 40 años, estos conflictos se han convertido en confrontaciones este-oeste y en guerras ideológicas, cuya resolución tropieza con el conocido artículo 2(7) de la Carta que dice "ninguna disposición de esta Carta autorizará a las Naciones Unidas a intervenir en los

\footnotetext{
${ }^{18}$ Sobre este tema, véase el extraordinario análisis de Éric de la Maisonncuve, La violence qui vient, (Arléa, 1997) y Edgar Morin; Sami Naïr, Une politique de civilisation, (Arlća, 1997).

${ }^{19}$ Richard J. Poncio, "Beyond 1995: negotiating a new UN through Article 109", Fletcher Fortum of World Affairs, 20(1):152, invierno/primavera de 1996.

${ }^{20}$ El Artículo 1, párrafo 3, cxpresa la necesidad de "lograr la cooperación internacional para solucionar problemas de carácter económico, social, cultural o humanitario".

${ }^{21}$ Ghassan Salamé, Appels d'empire: ingérence et résistances à l'âge de la mondialisation, 1996, p. 87.

${ }^{22}$ Edgar Morin, op. cit., p. 25.

${ }^{3}$ Eric de la Maisonnneuve, op. cit., p. 19.

${ }^{24}$ Eric de la Maisonncuvc, op. cit., p. 215, citando a Jcan Gurrtos (La pensée el la guerre, 1969).
} 
asuntos que son esencialmente de la jurisdicción interna de los Estados, ni obligará a los Miembros a someter dichos asuntos a procedimientos de arreglo conforme a la presente Carta". El predominio actual de los conflictos intraestatales o civiles va acompañado de formas de violencia difusa-proliferación de milicias (que lleva a la privatización de la violencia), criminalización de la política, masacre de civiles, terrorismo, genocidio $^{25}$ - que hacen que todos los esquemas analíticos y las relaciones interestatales resulten confusos. En estos conflictos 'en descomposición', 'degenerados' o 'anárquicos' ya no hay reglas, sino que se están convirtiendo en una violencia difusa. Aunque la violencia ocurra dentro de un país ya no concierne solamente a ese país, sino que atrae la atención del mundo entero. Se cuestiona la relación entre soberanía nacional y respeto por los derechos humanos. La 'intervención humanitaria' llevada a cabo por la OTAN en Kosovo aparentemente minó el sacrosanto principio de la soberanía nacional. Sin embargo, los criterios adoptados para la intervención no se aplican a cada conflicto o crisis del mundo y la 'comunidad internacional' todavía interviene demasiado tarde, como en Timor del Este, cuando ya han ocurrido las masacres.

Las reticencias a la hora de intervenir se deben a la estrecha concepción estatal de la soberanía y al hecho de que su contenido se ha mantenido igual desde el fin de la Segunda Guerra Mundial, mientras que el concepto en sí ha evolucionado. El concepto se ha alterado porque hoy día hay numerosos actores (supranacionales, transnacionales o subnacionales) y fuerzas (económicas, comerciales, tecnológicas y culturales) que contestan la soberanía estatal. Incluso, los estados se ven cada vez más amenazados por el creciente papel de los individuos, que está llevando a una soberanía popular. Existe una tendencia actual hacia la cesión o distensión de las prerrogativas de soberanía tradicionales de los estados nación a través de las organizaciones regionales (como en Europa) o a través del creciente cuerpo de leyes, convenciones y tratados internacionales sobre una amplia gama de temas. Además, como resaltó Samuel A. Makinda, la idea de soberanía ya no sólo hace referencia a la soberanía estatal sino también a la soberanía popular ${ }^{26}$, de la misma manera que la seguridad internacional va mano a mano con la 'seguridad humana'. De hecho, ya hace varios años que el Consejo de Seguridad tiene en cuenta la ampliación del concepto de seguridad inter-

${ }^{25}$ Pierre Hassner, "Par delà la guerre et la paix: violence ct intervention après la guerre froide", Entes, 1996.

${ }^{26}$ Samuel A. Makinda, "Sovereignty and International Security: Challenges for the United Nations", Global Govemance, 2(2):149-168, mayo-agosto de 1996. 
nacional y reconoce que "las fuentes no militares de inestabilidad en la esfera económica, social, humanitaria y ecológica se han convertido en amenazas a la paz y a la seguridad"27. Sin embargo, esto todavía no es algo sistemático sino que se aplica día a día y todavía está demasiado condicionado por los intereses contradictorios de los miembros permanentes del Consejo de Seguridad y de los grupos regionales.

No obstante, "una excesiva flexibilidad, una falta de clasificación y un enfoque 'pragmático', caso por caso, puede conducir a una 'incertidumbre operativa' y la no conformidad"28 y por tanto a un alejamiento de los principios en los que se basa la ONU. Esto nos obliga a cuestionarnos los objetivos iniciales de la Carta: ¿pretenden proteger a los estados o a sus ciudadanos ? $^{29}$ Más importante aún es el hecho de que la falta de rigurosidad a la hora de realizar estos cambios puede hacer creer a las personas que la ONU actúa de acuerdo con el principio de doble rasero. Por tanto, "a fuerza de ser demasiado selectivo a la hora de elegir sus misiones, el Consejo de Seguridad se puede convertir -si no lo es ya- en un órgano interestatal que trata asuntos que las grandes potencias no consideran importantes" ${ }^{\prime 30}$. De nuevo, en este caso, las decisiones del Consejo de Seguridad sólo reflejan la voluntad o falta de voluntad y los intereses ofalta de intereses de sus miembros, en lugar de reflejar aquellos de un órgano de concertación creado para implementar políticas cooperativas para el beneficio común.

Para el ejercicio eficaz de la soberanía popular a nivel internacional, multilateral o incluso regional, es necesaria la construcción de una 'sociedad civil internacional' mejor organizada y estructurada que la que existe actualmente. Durante las grandes conferencias de la ONU (especialmente durante la Cumbre Habitat II en Estambul ${ }^{31}$ ) ha surgido un embrión de sociedad civil internacional. En ellas participaron ONG, asociaciones, el sector privado, representantes de las comunidades locales, comunidades científicas y expertos. Lo mismo ocurrió en mayo de 2000 con el 'Foro del Milenio' que agrupó a representantes de ONG y otros

\footnotetext{
${ }^{27}$ Declaración del presidente del Consejo de Seguridad de las Naciones Unidas, 31 de cnero de 1992. 1995.

${ }^{28}$ Stanley Hoffmann, "Thoughts on the UN at Fifty", European Joumal of International Law, 6(3):321,

"Al referirse a la "tragedia argelina", Georges Kicjman considera que "la razón de ser de la ONU" no es proteger a las naciones, sino "a los hombres, mujeren y niños que las constituyen". "Le drame algérien et la Charte des Nations Unies", Le Monde, 13 de enero de 1998.

${ }^{30}$ Ghassan Salamé, op. cit., p. 150.

${ }^{31}$ Durante esta Cumbre, autoridades locales, organizaciones no gubernamentales y representantes del sector privado crearon foros consultivos "cuyas conclusiones (fueron) el sujeto de informes y recomendaciones que seguramente tuvieron un impacto directo en las negociaciones". Véase el artículo de Jérôme Bindé, "The City Summit: The Lessons of Istanbul", Futures, 29(3):218, 1997.
} 
grupos de la sociedad civil que hicieron propuestas para la 'Cumbre del Milenio' y trataron de crear una estructura organizativa en la cual las personas del mundo pudieran participar eficazmente en el proceso global de toma de decisiones. La conferencia de la Organización Mundial del Comercio mantenida en Seattle también ha mostrado el poder de una posible sociedad civil internacional organizada.

La cuestión es si esta sociedad civil es capaz de evaluar de manera eficaz y coherente las decisiones y la actuación de los estados. Lo único que se puede afirmar es que la participación de los actores de la sociedad civil en los debates y en el trabajo de la ONU puede, hasta cierto punto, ayudar a contener el poder de los principales estados y a reducir las tensiones entre la universalidad y la soberanía nacional. El problema es que la integración de los actores no estatales sigue siendo imperfecta y desigual, y sigue estando sometida a la buena voluntad de los estados, sobre todo en los órganos importantes ${ }^{32}$. Lo que ocurre con las organizaciones no gubernamentales también ocurre con los pequeños estados. Por ejemplo ¿se respetan siempre las disposiciones de los artículos 31 y 32 sobre la participación de los estados no miembros del Consejo de Seguridad en los debates? $Y$ aunque esta participación sea real iInfluyen realmente las ideas propuestas por estos estados sobre las 'predecisiones', tomadas casi siempre tras puertas cerradas por los miembros permanentes? En este caso, como en muchos otros, la aplicación rigurosa de los artículos de la Carta ya sería un paso hacia delante y reduciría la actitud instrumental que muestran las grandes potencias hacia la ONU.

Estos cambios internacionales crean tensiones que son difíciles de controlar. También constituyen desafíos clave a los que hay que hacer frente a los albores del siglo XXI. También ponen de manifiesto la diversidad de fuerzas y actores implicados en la esfera internacional y las paradojas estructurales que comportan. Existen tensiones entre el nivel transnacional y el nivel interestatal; entre soberanía e interferencia; entre intereses contradictorios; entre la lentitud de los estados y la rapidez de los otros actores; entre lo práctico y las visiones y las esperanzas; entre fuerza y poder por un lado y justicia e igualdad por otro; entre universalidad e individualismo o particularismos; y entre intervención, neutralidad e imparcialidad ${ }^{33}$. Es dentro de este contexto de tensiones y

${ }^{32}$ Por ejemplo, la Subcomisión de Prevención de Discriminación y Protección de las Minorias (órgano subsidiario de la Comisión de Derechos Humanos) presta la sala primero a las organizaciones no gubernamentales.

${ }^{33}$ Bruce Russett, "Ten Baiances for Weighing UN Reform Proposals", Political Science Quarterly, 111(2):259-269, verano de 1996. 
adaptación que se cuestiona el papel y la utilidad de las Naciones Unidas. La Organización mundial debería poder facilitar esta adaptación ejerciendo su papel de mediador y de regulador y proporcionando un vínculo entre todos los actores del sistema internacional. Debería fomentar una 'mediación social' que expresara el 'Nosotros universal' y "disolviera el 'Yo' en el ámbito internacional"34. Por tanto, la reforma de la organización mundial requiere tanto una adaptación estructural que le dé una mayor eficiencia y racionalidad como una adaptación conceptual que dé sentido a un proyecto colectivo.

\section{Una reforma estructural}

Desde sus comienzos, han predominado tres tendencias de pensamiento: una tendencia reformista que desea una 'revitalización' o una 'racionalización' de la Organización dentro del marco de la Carta; una tendencia más hostil que desea que la ONU se reduzca a mínimos (tendencia que hoy día representa el sector antiONU del partido Republicano norteamericano, y en particular el Presidente de la Comisión de Relaciones Exteriores del Senado, el Senador Jesse Helms ${ }^{35}$ ); una tendencia radical que pide la sustitución de la actual ONU por una 'Organización de Tercera Generación' (argumento apoyado por Maurice Bertrand) ${ }^{36}$. Estas tendencias tienen diversos impactos, pero todas ellas se plantean el problema de la adaptación de la Organización a los nuevos retos globales y al contexto internacional de principios del siglo XXI. ¿Cómo debe transformarse la ONU para no parecer una organización del pasado y para ser acorde con las aspiraciones de la población y con su margen de actuación? La respuesta a estas preguntas debe basarse en los conocimientos y en una visión realista de la naturaleza y de los logros del sistema de las Naciones Unidas.

A las personas críticas que opinan que la ONU es inútil hay que recordarles que la Organización ha obtenido grandes logros. Basta mencionar su actuación a favor de la ampliación del derecho internacional, de la deslegitimación de la guerra entre estados, de unas mejores relaciones interestatales mediante la diplomacia multilateral y sus logros en

\footnotetext{
${ }^{34}$ Zaki Laïdi, "La mondialisation tuc-t-clie l'universei?", Sources UNESCO, $\mathrm{N}^{\circ} 79$, mayo de 1996.

${ }^{35}$ Véase su artículo: "Saving the UN: A Challenge to the Next Secretary-General", Foreign Affairs, 75(5):27, septiembre/octubre de 1996.

${ }^{36}$ Véase Maurice Bertrand y Daniel Warner (cds.), A New Charter for a Worldwide Organization, (La Haya, Martinus Nijhoff, 1996), 288 pp.
} 
materia de derechos de los más pobres y derechos humanos en general (en su sentido más amplio: la promoción de los derechos de los niños, de las mujeres, de las minorías de los indígenas y de los refugiados). Es evidente que la Organización no puede ser responsable de la indecisión de sus estados miembros, ni de su falta de voluntad política y de sus errores.

Recordemos también que la ONU no es un actor independiente o autónomo en relaciones internacionales como los estados: la supranacionalidad que se otorga a sí misma, o que se le otorga, es en realidad sólo teórica. Las Naciones Unidas no son un gobierno mundial, sino un sistema de cooperación entre estados. Es una organización intergubernamental cuyo poder de decisión está en manos de los estados miembros y sobre todo en manos de los más poderosos. Por consiguiente, la Organización no tiene recursos financieros propios, sino un presupuesto formado por las contribuciones de sus miembros, y el cabeza de la Organización, el Secretario General, lo nomina el Consejo de Seguridad y luego lo nombra la Asamblea General. Ghassan Salamé resume de manera clara y exacta la situación: "la ONU es la hija de los estados, pero una hija no querida. Aunque se le han dado los instrumentos para actuar, se considera que no merece utilizarlos. Se le encomienda mantener la paz pero debe actuar bajo estricta observación de la 'competencia nacional' de sus miembros. Se le acusa de ser pasiva, ineficaz o contraproducente pero se le niegan los medios económicos para realizar su misión. Peor todavía: las grandes potencias la consideran universal pero le prohíben meterse en los problemas que les conciernen de cerca y que amenazan a la seguridad internacional. Es por eso que las críticas hacia la ONU no suenan convincentes...: más que una declaración objetiva son una acusación de un organismo que no quiere - o no puede- acometer el objetivo principal que le han asignado los poderosos, es decir, encubrir las dudas, las contradicciones o simplemente la cobardía de los gobiernos"37.

Es necesario distinguir entre la naturaleza de la Organización y sus funciones. Las funciones de la Organización a veces la hacen parecer algo más que la simple suma de sus componentes nacionales y desempeñar un papel 'semiautónomo'. Esto depende del área en la que actúe y de los intereses que amenace. Los estados le añaden funciones de vez en cuando: un instrumento de política exterior, un foro de negociación, un chivo expiatorio, un órgano de legitimación. Pero, en cualquier caso, la

${ }^{37}$ Ghassan Salamé, op. cit., pp. 137-138. 
Organización tiene una independencia relativa y limitada por la soberanía de los estados y por los intereses nacionales. Las decisiones, actuaciones o inercia de la Organización son el resultado de las luchas de poder y los conflictos de intereses que tienen lugar en el sistema internacional y que se reproducen en el seno de la Organización. Esto explica en parte la lentitud del proceso de reforma, que debe tener en cuenta la opinión, intereses y propuestas de todos los estados miembros (189) y de los grupos regionales ${ }^{38}$.

Hoy día prevalece la tendencia reformista, debido a que lo radical de las otras dos tendencias implicaría transformaciones demasiado importantes. Existiría en teoría una cuarta tendencia apoyada por algunas ONG: la que apoya la supranacionalidad efectiva de las NN. UU. Pero los estados todavía no están preparados para tolerar la existencia de un verdadero actor supranacional que limitara de modo más abierto y eficaz su poder y libertad de decisión. Una dinámica de cambios no implica necesariamente un proceso revolucionario, sino que puede darse un proceso de evolución y adaptación ${ }^{39}$. Por consiguiente, ante la cuestión de la adaptación de la Organización a su entorno, los miembros han mostrado claramente una voluntad de racionalizar la Organización, de renovarla sin cambiar sus fundamentos. Por tanto, sin ser capaces de llegar a un consenso real sobre el futuro de la ONU y sobre cómo entender el papel de una organización internacional, en estos cuatro años, los estados han establecido una serie de prioridades que deberían inspirar el proceso de reformas. Este proceso debería consistir principalmente en la 'limpieza' del sistema, basada en identificar sus ventajas comparativas, establecer una mejor coordinación entre las agencias y encontrar la voluntad de hacerlo mejor con menos. En la "Cumbre del Milenio", los cinco miembros permanentes del Consejo de Seguridad han identificado las siguientes "áreas prioritarias": reforzar el liderazgo de la ONU en materia de paz y seguridad; reforzar el mantenimiento de la paz; revitalizar la gestión; completar los recursos humanos; reafirmar el compromiso financiero ${ }^{40}$.

\footnotetext{
${ }^{38}$ James Paul lo resume de la siguiente manera "la gente está en desacuerdo sobre el tipo de reforma que requiere la ONU y con el propósito. Los líderes de ONG quieren una ONU más democrática, más abierta y transparente. Los tecnócratas buscan mayor productividad y eficiencia por parte del personal de la ONU. Los delegados apoyan reformas que favorezean los intereses nacionales y el poder nacional. Los idealistas ofrecen planes para un ente ampliado que reduzca la soberania de los estados. Micntras que los conservadores apoyan una ONU reducida con menos poder. Es extremadamente difícil llegar a un acuerdo." En: "UN Reform: An Analysis", Global Policy Forum, http://www.globalpolicy.org/reform/analysis.htm, 1 de abril de 1998.

${ }^{39}$ Keith Krause y Andy Knight ("Evolution and Change in the UN System", p. 12), piensan que la evolución del sistema internacional puede consicerarse un proceso dialectico, en State, Sociery, and the UN System: Changing perspectives on multilateralism, (UNU Press, 1995).

${ }^{40}$ Déclaration du Sommet du Conscil de Sćcurité des Nations Unies (A/RES/55/2), 7 de septiembre de 2000.
} 
Según los miembros del G7/G8, para ser más eficiente, la ONU debe "aclarar su papel y sus ventajas comparativas. Debe aumentar la eficacia de la Secretaría y del marco operativo, hacerlos más coherentes y asegurar una coordinación genuina a todos los niveles" ${ }^{\prime 24}$. También esperaban que la ONU se centrara más en sus actividades de desarrollo. Ahora se dan cuenta de que la ONU no puede abandonar sus actividades de mantenimiento de la paz. El informe Brahimi esboza estas orientaciones y a la vez insta a que se hagan reformas en este campo ${ }^{42}$. Tras los fracasos de la ONU en Somalia, Bosnia y Ruanda ('fracasos' que se debieron principalmente a la falta de voluntad y estrategia política de los grandes miembros de la ONU), los estados ya no se fiaban de la ONU y preferían actuar a través de coaliciones multilaterales u organizaciones regionales. Entre el 1995 y el 2000, la ONU se ha convertido en una Organización que mantiene el orden, en lugar de mantener la paz, y las 'operaciones de mantenimiento de la paz' son en realidad misiones policiales o administrativas que reconstruyen las estructuras administrativas de un estado. Hoy día, con las deficiencias del mantenimiento de la paz de la ONU en Sierra Leona y en el Congo, los estados parecen dispuestos a racionalizar las actividades de mantenimiento de la paz y a reestructurar el Departamento de Operaciones de Mantenimiento de la Paz. Son necesarias sobre todo reformas en materia de planificación, despliegue rápido, reglas de combate, equipamiento de las tropas y análisis de la situación sobre el terreno. En estos campos, la implementación de la mayoría de las recomendaciones del informe Brahimi mejoraría la gestión de las operaciones de mantenimiento de la paz desde la Sede. Sin embargo, los estados deberían ir más lejos y adaptar el mantenimiento de la paz a las nuevas características de los conflictos. Se debería pensar más acerca del uso de la fuerza, de la coerción y de la imposición de la paz: todos estos temas están en juego en las actuales operaciones de paz.

Con el fin de racionalizar la actuación del sistema de Naciones Unidas, los miembros desean una mayor coordinación entre los diversos componentes del sistema de la ONU, reforzar la cooperación y "una mayor coherencia en sus políticas (...) a fin de seguir un camino coordinado hacia los temas de paz y desarrollo"43. En momentos de crisis financiera y restricciones presupuestarias, los estados quieren que la ONU lo haga mejor con menos. Esto significa hacer retoques, solucio-

4! Párrafo 42 del G7 Economic Communiqué en Lyons, junio de 1996.

42 Informe Brahimi (A/55/305 - S/2000/809, 21 de agosto de 2000).

${ }^{43}$ Informe del Milenio, 8 de septiembre de 2000. 
nar el hecho de que coincidan o se dupliquen las competencias, eliminar o 'fusionar' algunos fondos o programas que son redundantes entre sí o con las agencias especializadas ${ }^{44}$. Aquí parece que se reconoce el rol y las competencias de las agencias especializadas. Sin duda, es indispensable que las distintas agencias especializadas del sistema recuperen toda su autoridad frente a los fondos y programas que casi siempre invaden sus competencias, lo que conduce a una hipertrofia artificial de todo el sistema de Naciones Unidas. No obstante, desde la cumbre del G8 en Denver, el papel de las agencias especializadas se cuestiona más o menos tras la exigencia de "un examen urgente y en profundidad de los fondos y programas de la ONU, además de un examen global del sistema y del papel y mandato de las agencias y comisiones especializadas". El Secretario General respondió a estas exigencias en julio de 1997 recomendando la creación de una 'Comisión Especial' con ese propósito. Todavía está pendiente la creación de esta Comisión. De cualquier modo, los estados no especificaron qué dirección debía tomar este 'revisión': ¿una recentralización del sistema bajo el auspicio del PNUD y las instituciones de Bretton Woods, o una división del trabajo y una verdadera redistribución de las tareas ${ }^{45}$ ? Pero para ser realmente eficaz y completa, la reforma también debería implicar a las instituciones de Bretton Woods que deberían centrar su trabajo en los aspectos financieros de la ayuda al desarrollo y dejar de interferir en las principales áreas de las instituciones especializadas del sistema de Naciones Unidas.

Un mecanismo de cooperación verdadero y eficaz debería armonizar las actividades de todos los componentes del sistema de la ONU para que estas instituciones no adopten políticas o programas contradictorios como ha ocurrido en el pasado ${ }^{46}$. Esto implicaría un cambio radical de las estructuras del sistema e innovaciones en las relaciones entre las distintas agencias. Esto es especialmente cierto si, como dice James Paul, tenemos en cuenta que los inconvenientes del sistema de la ONU son el resultado de "un profundo desacuerdo político entre los miembros

${ }^{44}$ Por ejempio, el Programa Mundial de Alimento y el Fondo Internacional de Desarrollo Agrícola compiten con la FAO; la Comisión de Desanollo Sostenible compite con el Programa de las Naciones Unidas para el Medio Ambiente; UNICEF, el Banco Mundial y el PNUD compiten con la UNESCO en ei campo de la cducación.

ts En cualquier caso, dicha reforma seria muy difícil de implementar ya que los lideres de las agencias especializadas no son diputados del Secretario General de la ONU, sino que son líderes de órganos independientes.

${ }^{46}$ Por cjemplo, durante mucho ticmpo, las políticas de ajuste estructural impuestas por cl FMI y cl Banco Mundial no tenían en cuenta las políticas sociales recomendadas por la OIT, UNICEF, la FAO o la UNESCO. Esto tambiên es debido a la "verticalidad" del sistema que hace que cada institución sea independiente. Véase el articulo de Alain Destexhe, "L'ONU au chevet du monde", Politique intemationale, verano de 1993, № 60, pp. 195-208. 
y entre otras fuerzas antagónicas ${ }^{\prime 47}$. Sin embargo, este tipo de coordinación sólo es posible si existe una coordinación a nivel estatal, ya que la 'micro coordinación' no puede resultar efectiva sin una coordinación a grande escala y sin un consenso sobre los objetivos a alcanzar. En el proceso de toma de decisiones, la coordinación debe hacerse a tres niveles diferentes: entre estados, entre estados y las instituciones del sistema y dentro de los estados, entre sus distinto órganos gubernamentales.

Finalmente, se hace especial énfasis en la reforma financiera ${ }^{48}$ : cómo modificar las contribuciones de los estados y cómo reducir el presupuesto de la ONU. Sobre todo los Estados Unidos quieren obtener, durante la quincuagésima quinta sesión de la Asamblea General de la ONU, una reducción de su contribución al presupuesto para el mantenimiento de la paz de un 31 a un $25 \%$ y de su contribución al presupuesto general de un 25 a un 22\% (y si es posible a un 20\%). Ya se han hecho grandes recortes en la mayoría de las agencias, fondos y programas del sistema de Naciones Unidas (sobre todo en la UNCTAD y ONUDI). Desde 1995, el presupuesto de la ONU se recorta regularmente. Los Estados Unidos se oponen a cualquier incremento del presupuesto de la ONU. Esta política es contradictoria al hecho de que a veces la implementación de las reformas requiere financiación.

El impago de las contribuciones es un problema completamente político y no financiero. Comparado con el presupuesto de una gran potencia, el presupuesto de la ONU US $\$ 1.3$ billones para el presupuesto ordinario y US $\$ 3$ billones para el presupuesto de mantenimientos de la paz) es extremadamente bajo. El presupuesto para el mantenimiento de la paz representa el $1.1 \%$ del presupuesto militar de Estados Unidos, lo que equivale a dos días de operación "Tormenta del desierto". El presupuesto ordinario de la ONU representa aproximadamente un $4 \%$ del presupuesto anual de una ciudad como Nueva York ${ }^{49}$. La actual contribución de los Estados Unidos al presupuesto ordinario le cuesta a cada americano US\$1.2 al año y el de mantenimiento de la paz unos US\$ 7 Sin embargo, quizá sean más significativos los US\$ 5 billones que emplea el sistema de la ONU en

\footnotetext{
${ }^{47}$ James Paul, "UN Reform: An Analysis", op. cit. (nota 38). S. Cortembert subraya, "por qucrer un sistema descentralizado, se ha creado un nuevo órgano cada vez que ha surgido una nueva cucstión internacional. Por tanto, las responsabilidades internacionales se han repartido cntre numerosos órganos importantes". Taller en la University of Besançon, L'ONU, 50 ans après: bilan et perspectives, $29-30$ de marzo de 1995, p. 44.

* Las propuestas para que ia Organización contara con recursos financieros independientes se han dejado fuera de la agenda de negociación. Por cjemplo, la 'Tasa Tobin' (impuesto sobre las transaccioncs financicras). Véasc PNUD Inforne sobre el Desarrollo Humano 1994, p. 75. Se han hecho otras propuestas en el capítulo 5 del informe Ramphal/Carisson (impuesto sobre billetes de avión, tráfico marino, ctc.)

${ }^{* 9}$ Información proporcionada por el Bulletin du Centre d'information des Nations Unies à Paris, $N^{\circ} 19$, mayo de 1996, p. 38.
} 
el campo económico y social, que equivale a un gasto de 88 centavos por cada habitante del planeta; aunque a la vez los estados se gastan US $\$ 767$ billones por año en material militar lo que equivale a US\$ 134 por habitante $^{50}$. Esto es mucho más, según Wally N'Dow, el Secretario General de la Cumbre de la Ciudad en Estambul, de lo que se necesita (menos de US $\$ 100$ por persona) para "dar un techo, agua limpia y servicios sanitarios a cada hombre, mujer y niño del planeta"s!.

Sin duda estas micro reformas son muy útiles, porque permitirían que el enormemente descentralizado sistema de la ONU se convirtiera en un sistema más 'compacto' y más racional con unas líneas de autoridad más nítidas. También conseguirían un mayor equilibrio entre los componentes del sistema. Pero aquí, como en cualquier otro sitio, los estados todavía tienen que demostrar su determinación y voluntad de implementar estas propuestas que requieren un cambio de comportamiento y que ponen en cuestión algunos intereses. Los diversos grupos de trabajo todavía no han llegado a un acuerdo sobre propuestas concretas y sobre un calendario y unas medidas exactas. Sin embargo, aunque se justifiquen estas propuestas, no pueden constituir un verdadero proyecto de futuro y no son suficientes. Las reformas eventuales u organizativas nunca podrán sustituir a las reformas en profundidad que combinan visión y proyecto o estrategia a largo plazo, a fin de restablecer la credibilidad de las acciones y decisiones de la ONU. No sólo debemos preguntarnos el 'cómo' sino también 'qué hacer' y 'cuáles deberían ser las misiones de la ONU'.

\section{Hacia una reforma conceptual}

Parece cada vez más que para ser eficaz y creíble, la ONU debe centrarse en lo que hace mejor y en lo que realmente puede hacer. Esto significa que debería aprovechar al máximo el intersticio que dejan sus miembros. Realmente es "en los intersticios del interestado, dominado, como siempre, por las desigualdades y las rivalidades, donde amanecen tímidamente los elementos de una conciencia, competencia y solidaridad universal, o al menos universalista"52. Ahora, la ONU debe recuperar una acción, un método de funcionamiento y un liderazgo coherentes y coordinados, cuyo denominador común sea la previsión y la prevención.

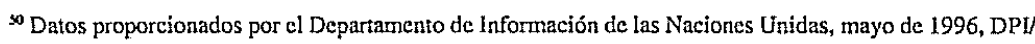
1753/Rev.3.

sl Citado por Jćrôme Binde, op. cit., p. 226.

52 Pierre Hassner, 1996.
} 
Estos dos objetivos no tieneN por qué obligar a la ONU a abandonar sus actividades de mantenimiento de la paz, al contrario, debería reforzar$\operatorname{las}^{53}$. Aquí proponemos tres posibilidades de reforma de Naciones Unidas para que la Organización acompañe a las evoluciones internacionales; para que actúe en lugar de reaccionar. Una acción reforzada con el objetivo de explotar las ventajas comparativas del sistema de Naciones Unidas, una gestión renovada centrada en una ética de trabajo que conduzca a la realización de un proyecto colectivo y una liderazgo fuerte que beneficie a la comunidad internacional: éstas son las orientaciones de reforma modestamente propuestas aquí.

\section{Una actuación reforzada}

La actuación de la ONU debería estar gobernada por el siguiente trípode: desarrollo sostenible / prevención / cultura de paz. Este trípode debería integrarse en una amplia concepción de la seguridad internacional y en una visión a largo plazo. Como dijo Boutros Boutros-Ghali, la ONU debe desarollar "una acción preventiva para controlar mejor el presente y una acción con perspectiva para enfrentarse mejor al futuro" 54 .

El desarrollo es la base más segura para la paz: también es "la tarea más importante a la que se enfrenta la humanidad hoy en día"s5. El desarrollo sostenible es "el desarrollo que satisface las necesidades del presente sin comprometer la capacidad de las generaciones futuras para satisfacer sus propias necesidades"s6. Este desarrollo debe centrarse ante todo en el ser humano. También debe respetar el medio ambiente y reconocerse como un derecho humano fundamental. Por lo tanto debe garantizarse a través de una comprensión intelectual más amplia, un compromiso moral más profundo y unas medidas políticas más eficaces. Los gobiemos de los países más ricos están descubriendo que las medidas económicas no son la solución ${ }^{57}$ y que deben hacerse algunos esfuerzos

\footnotetext{
${ }^{53}$ No obstante, en este tema, cualquier reforma de los métodos de acción debería cstar precedida por una reflexión sobre el uso de la fuerza de las Naciones Unidas sobre el terreno, en caso de no cooperación entre las partes. Véase la tesis doctoral de la autora: Ability and Inability of the UN Security Council to Use Force - Bases, Practice, Perspectives, diciembre de 1999, Université Panthéon-Assas (París 2), 966 pp.

${ }^{\text {sa }}$ Boutros Boutros-Ghali, "Le Secrétairc général des Nations Unies: entre l'urgence et la durée", Politique étrangère, verano dc 1996 , p. 411.

${ }^{35}$ Boutros Boutros-Ghali, Un Programa de Desarrollo, 1995, §1.

s6 Palabras de Gro Harlem Brundtland, ex presidenta de la World Commission on Environment and Development. Citado por Brian Urquhart ("Lcarning from the Gulf", New York Review of Books, 38(5):36, 7 de marzo de 1991) que añade que "una de las preguntas más importantes de nuestros tiempos es si es alcanzable el desarrollo sostenible a escala mundial".

s7 Como dijo Burhan Ghalioun, "la competencia económica no puede constituir la razón de ser de una sociedad y tampoco puede darle sentido a la existencia humana", en "La déstabilisation du monde", Défense nationale, abril de 1996.
} 
por los países más pobres y por las regiones y clases sociales más pobres dentro de los países.

La Cumbre del G8 en Colonia expresó una voluntad de "profundizar un partenariado para el desarrollo" entre los países en desarrollo, los países desarrollados y las instituciones multilaterales. Un partenariado cuyo objetivo fuera el desarrollo sostenible y la erradicación de la pobreza, y que estuviera basado en el espíritu de la solidaridad porque "la paz en casa ... requiere paz en el extranjero y cooperación entre las naciones"58. Sin embargo, todavía queda mucho para que la ayuda oficial al desarrollo de los países industrializados alcance a ser el $0.7 \%$ de su $\mathrm{PNB}^{59}$; la ayuda oficial al desarrollo no puede reemplazarse únicamente por inversión privada, ya que es demasiado selectiva e implica demasiadas exigencias.

Además, el desarrollo no sólo significa mayor competitividad económica y tecnológica sino también, y sobre todo, un mayor bienestar para la población, como por ejemplo, mejores condiciones de vida, educación, condiciones sanitarias y mayor respeto por el medio ambiente. Por lo tanto, el sistema de Naciones Unidas debe implementar una estrategia de desarrollo coherente para lograr mayor coordinación entre las agencias - para ello, el Consejo Económico y Social debería revitalizarse para convertirse en un Consejo de Seguridad Económica que trabajara todos los temas de desarrollo y facilitara la cooperación- además de una mayor coordinación entre las instituciones y los estados o actores de este campo (ONG, asociaciones, organizaciones regionales) y luego ayudar a obtener un mejor resultado de los proyectos. Es más, "el desarrollo sostenible requiere una asociación firme entre las autoridades y la sociedad civil"60. Ante todo, estos proyectos deberían tener en cuenta los deseos de la población local y ayudarles a llevar a cabo y favorecer la emergencia de una sociedad civil. A la vez, estas personas deberían recibir todo lo que les sea necesario en términos de avances tecnológicos. Su desarrollo también debe incorporar restricciones debido a temas medioambientales (por ejemplo el problema del agua, la desertificación, la fertilidad del suelo y la urbanización). Los países del Norte deben compartir su progreso tecnológico, su experiencia y su 'pericia', que a su vez puede que ponga fin o reduzca la fuga de cerebros de los ciudadanos del Sur. En pocas palabras, es de gran importancia que los países más

\footnotetext{
${ }^{58}$ Un programa de paz, op. cit., párrafo 80.

${ }^{39}$ La ayuda püblica al desarrollo proporcionada por los países ricos representaba en 1997 sólo el 0,21\% de su PNB (44 mil miliones de dólares), que es la cifra más baja desde los años 50 .

${ }^{\circ} \mathrm{A} / 49 / 665,11$ de noviembre de $1994, \$ 17$.
} 
ricos ayuden a los países pobres a desarrollar su propio potencial económico, técnico y humano. Por lo tanto como dice Jean-Paul Marthoz, "el mundo no necesita tanto la intervención humanitaria como el reparto global"6l.

El segundo pilar de actuación de la ONU debe ser la prevención de las crisis y del conflicto. El desarrollo en sí es una forma de prevención. La diplomacia preventiva (mediante misiones de recogida de datos, el despliegue preventivo, los buenos oficios y la mediación) comporta otros métodos de prevención. Ambos transcurren en un amplio concepto de seguridad internacional. Como dijo un ex Secretario General de Naciones Unidas, "podemos prevenir los nuevos conflictos emergentes sólo con tener una concepción mucho más amplia y global de la propia noción de seguridad"62. La prevención no se reduce al control de armas, a la resolución pacífica de conflictos, al desarme (sobre todo prohibiendo el uso y la producción de minas antipersonales), sino que también implica una seguridad económica, social y cultural ${ }^{63}$. El informe Brahimi resalta que es necesario que la ONU mejore sus estrategias de prevención de conflictos.

La prevención está relacionada con poseer y analizar una información independiente e interdisciplinaria ${ }^{64}$, con un cambio radical en la manera de tratar incidentes, crisis y problemas. Se debe favorecer por un lado el largo plazo en lugar de la emergencia y por otro lado encontrar de nuevo "la capacidad de dar soluciones en situaciones de alerta" 65 y aprender a "invertir en lo intangible"66. El problema de la información es crucial para la ONU ya que la información es necesaria para tomar cualquier medida y porque el análisis de los datos gobierna su procesamiento. La información no sólo debe ser independiente sino lo más precisa, detallada y no dividida o fragmentada entre varios servicios, posible; y debe ser actualizada constantemente. La ONU no debe recibir solamente datos oficiales de los miembros, corriendo el riesgo de llegar a conclusiones poco meditadas o erróneas ${ }^{67}$. Todo lo contrario, debe realizar un análisis que tenga en cuenta todos los aspectos del problema, detectar todas sus

\footnotetext{
${ }^{61}$ Jcan-Paul Marthoz, "Everything has change, except ourselves", GRIP, octubre de 1995, p. 174.

${ }^{62}$ Boutros Boutros-Ghali, op. cit., p. 412.

${ }^{63}$ El embajador Hector Gross Espiell, añade que "sin este concepto global de seguridad, seguiremos en un marco estrecho que no es compatible con la realidad actual". Conferencia en la UNESCO, 25 de enero de 1996.

"Véase el artículo de Maurice Bertrand, "Vers une strałégie de prévention des confits", Politique étrangère, prinavera de 1997, pp. 111-123.

${ }^{65}$ Zaki Laîdi, "Le rite méđiatique du G7", Libération, 15 de junio de 1996.

${ }^{6}$ Federico Mayor, "A New Beginning", UNESCO Courier, noviembre de 1995, p. 7.

${ }^{67}$ Véase el articulo de François Moricoui-Ebrard, "Explosion urbainc, le sens de la démesure", Le Monde diplomatique, julio de 1996, p. 13.
} 
raíces y evitar los estereotipos, juicios de valor o etnocentrismos ${ }^{68}$. Esto es fundamental para garantizar la objetividad de la Organización, para desarrollar su función de experto y para dar el ímpetu decisivo a la creación de un sistema de aviso con tiempo. La recogida de información diversificada de varias fuentes y el llevar a cabo muchas misiones sobre el terreno permite comprender situaciones complejas y sociedades y por lo tanto actuar antes de que estalle un conflicto. Además, este método, basado en la proximidad, daría a las poblaciones una sensación de seguridad y daría credibilidad al trabajo de las instituciones de la ONU que estarían más cercanas a las preocupaciones e historia de la población.

Esta acción preventiva se desarrolla a largo plazo, anticipando; no tiene fin, sino que se evalúa constantemente y es innovadora. Debe tanto "conciliar valores universales y respeto por los particularismos"69 como promover la idea de progreso y a la vez combinar tradición y modemidad.

Si la prevención es el segundo aspecto de la construcción de la paz, la ampliación de la "cultura de paz" es el tercero. Además, es la medida más eficaz para combatir la 'cultura de violencia' que nos rodea, aunque también puede que sea el aspecto más difícil de implementar. La cultura de paz abarca comportamientos, prejuicios, falta de comunicación e intolerancia. Rechazar la 'cultura de violencia' significa desligitimizar las luchas de poder, el uso de la fuerza para resolver disputas y los comportamientos violentos. La 'cultura de paz' es la gestión no violenta de las crisis y conflictos; es la implementación de procedimientos democráticos y el respeto de los derechos humanos fundamentales; es la participación de todos los abogados de la sociedad en un diálogo constructivo. En pocas palabras "es la construcción de un marco de justicia, dignidad, igualdad y solidaridad"70. La cultura de paz es un concepto que tiene en cuenta el lugar de los individuos en el día a día hacia la construcción de la paz. De cierto modo es lo que Norbert Ropers denomina un "reto transnacional"71.

Para todas estas actividades, la ONU debe convertirse en un marco regulador internacional que sea una fuerza motriz, que concentre la energía alrededor de unas aspiraciones económicas, sociales y cultu-

\footnotetext{
${ }^{68}$ Para un anälisis pertinente de cstos problemas, véase Gérard Prunicr, "Manipulation humanitaire", Le Monde des débats, $\mathrm{N}^{\circ} 21$, julio-agosto de 1994, p. 5.

${ }^{69}$ Michel Wicviorka, Le Monde, 8 de octubre de 1996.

${ }^{70}$ Federico Mayor, "Fifty Years On", The UNESCO Courier, octubre de 1995, p. 6.

${ }^{71}$ Norbert Ropers, "The culture of peace and the promotion of a culture of constructive conflict management", julio de 1994.
} 
rales bien definidas y se gestione a un ritmo razonable. Desde esta perspectiva, la globalización de los intercambios comerciales, financieros, tecnológicos o culturales ya no supone un obstáculo o un factor de exclusión, sino un bien compartido por todos. Esta coordinación de las fuerzas de la globalización debe ir de la mano de la protección de la herencia humana cultural y global y de la preservación de su diversidad para una mayor tolerancia y una mejor integración. Estas acciones deben ser generadas por un funcionamiento adecuado de la administración de la ONU y por un liderazgo más autónomo. Participan en el reforzamiento de la construcción de la paz en todos sus aspectos.

\section{Una gestión renovada}

La gestión del sistema de Naciones Unidas debe estar a tono con su cultura de negociación y consenso. Por lo tanto, debe establecerse el diálogo, la consulta y las vías de comunicación a todos los niveles, entre todos los servicios, departamentos y actores (funcionarios, representantes, delegados, observadores). Rivalidad entre instituciones, servicios o personas debieran dar lugar a una cooperación que beneficie a todo el sistema. La eficacia también requiere un sistema de evaluación donde los resultados realmente se tengan en cuenta a la hora de mejorar los programas, proyectos o acciones actuales. Además, el reclutamiento de personal debe hacerse según la competencia de los candidatos y no debe estar sujeto a las presiones de los estados miembro. Por lo tanto, la gestión del sistema debe estar determinada por tres prioridades: pericia, formación y planificación a largo plazo.

Cada funcionario de la ONU debe ser considerado un experto en su campo de estudio y sus conclusiones independientes y científicas deben ser respetadas por los estados y otros actores. Al funcionario se le debe permitir que ejerza un liderazgo real a la hora de orientar o elegir políticas.

En segundo lugar, la ONU debe ser un centro de reflexión, una escuela de enseñanza de la no violencia, el diálogo intercultural y entre sociedades, el respeto por las diferencias. Esta enseñanza se haría a través de micro proyectos emprendidos en asociación con ONG u otras asociaciones, o a través de la cooperación de las instituciones especializadas del sistema de la ONU. 
En último lugar, la ONU no debe dejarse llevar por la tentación de responder a situaciones de emergencia ${ }^{72}$. Sin lugar a duda su papel se desarrolla en el largo plazo, "proteger a las generaciones futuras del flagelo de la guerra"73. Debe ser capaz de prever las necesidades humanas, las consecuencias negativas del deterioro del medio ambiente, los problemas que genera la distribución desigual de la riqueza y de los recursos naturales. Por eso parece indispensable la creación de una unidad de previsión (que sea parte del ejecutivo del Secretario General) tal y como existen en numerosas otras instituciones internacionales (la Oficina de Análisis y Previsión de la UNESCO, el Forward Studies Unit de la Comisión Europea, el Programa de Estudio del Futuro a Largo Plazo de la OCDE). Podría alertar a la opinión pública de los retos venideros y de los medios para resolverlos antes de que éstos se produzcan. Debemos por tanto alegrarnos de la creación, hace tres años, de una 'Unidad de Planificación Estratégica’. Esta Unidad debe ser reforzada y se le debe prestar más atención. Es más, tal y como ha recomendado el informe Brahimi, debería crearse una unidad de Información y Análisis estratégico a nivel del Departamento de Asuntos Políticos y el Departamento de Mantenimiento de la Paz, a fin de avisar sobre los primeros síntomas de una crisis. Es la única manera de que la Organización Mundial sea capaz de recuperar una actitud innovadora, un papel activo e incluso previsor y por lo tanto reafirmarse como una institución pacífica y reguladora. Este es el papel que debería desempeñar el Secretario General de la ONU.

\section{Un liderazgo reconocido}

Últimamente, muchos miembros han querido reducir el papel del Secretario General al de un simple gestor administrativo. Pero, según las disposiciones de los Artículos 97, 98 y 99 de la Carta, el Secretario General no es sólo el 'más alto funcionario administrativo' de la Organización sino que también, y principalmente, es un participante político que posee un verdadero poder de iniciativa.

\footnotetext{
${ }^{72}$ Esto no significa que las Naciones Unidas deba dejar de lado las situaciones que requieren acción urgente (epidemias, desplazamientos de poblaciones...). Para estos casos, la Organización debería tener también capacidad de reacción rápida, sobre todo en el campo humanitario. Sin embargo, la previsión y la acción preventiva deberian reducir estas acciones a situaciones de emergencia.

${ }^{73}$ Cita de la carta constitutiva de la UNESCO.
} 
El Artículo 98 le da el derecho de asistir a "todas las reuniones de la Asamblea General, del Consejo de Seguridad, del Consejo Económico y Social y del Consejo de Administración Fiduciaria", de participar en el trabajo de otros órganos y también el poder de influir en su agenda, poniendo en ella todas las cuestiones que considere deban ser discutidas. El Artículo 99 da al Secretario General el poder de iniciativa diplomática y un poder discrecional a la hora de decidir si traer un asunto ante el Consejo de Seguridad o no. Estas disposiciones requieren un juicio personal y una opción política y, al menos, le permiten hacer sugerencias ${ }^{74}$. Las frases 'en su opinión' y 'puede poner en peligro' del Artículo 99 permiten al Secretario General conducir acciones preventivas o de anticipación. La reciente crisis entre Irak y Estados Unidos ha demostrado que el Secretario General puede constituir una 'tercera vía', un buen intermediario o moderador.

Es aquí donde debe destacar el papel y función del Secretario General: prever, denunciar, alertar. Se espera que denuncie las violaciones de derechos humanos en cualquier país (haya las presiones que haya), que avise de las consecuencias de determinadas políticas, que prevenga de los conflictos que pueden emerger y destacar lo inadecuado de ciertas acciones. En pocas palabras, como dijo ciertamente Javier Pérez de Cuellar, el Secretario General es y debe ser la 'consciencia' de toda la humanidad: "el Secretario General debe pedir el desarme, la tolerancia y la solidaridad en nombre de las personas"75. En ese sentido, está en sus manos promover algún tipo de ética a nivel internacional y ser el protector de los menos poderosos. Siendo una autoridad moral, el Secretario General tiene más poder para influir en el comportamiento de los estados, para conseguir que respeten las condiciones de la Carta de principios de la ONU a la que se adhirieron (aunque los resultados de esta influencia no sean siempre visibles a corto plazo). Kofi Annan dijo "si no hablamos en voz alta, de forma individual y colectiva, hoy y siempre que nuestra consciencia se enfrente a la inhumanidad y a la intolerancia, no habremos cumplido con nuestro deber - hacia nosotros mismos y hacia las generaciones futuras-"76. La función de la información del Secretario General es esencial en este sentido. La información que posee le

\footnotetext{
${ }^{74} \mathrm{El}$ artículo 99 se invocó formalmente en tres ocasiones: durante la crisis del Congo en julio de 1960 (Dag Hammarskjöld), durante el episodio de los rehenes americanos en Teherán en noviembre de 1979 (Kurt Waldheim) y en relación con la situación en el Líbano en 1989 (Javier Pérez de Cuellar).

7 Javier Pérez de Cuellar, "Le rôle du Secrétaire génćral des Nations Unies", Revue générale de droit international public, $\mathrm{N}^{\circ} 2,1985$.

${ }^{76}$ Deciaración deI cuadragésimo cuarto período de sesiones de la Comisión de Derechos Humanos, 16 de marzo de 1998, Gincbra, SG/SM/98/53.
} 
permite, de manera independiente, anticipar acontecimientos, 'mediatizar' un problema o sugerir una serie de soluciones.

\section{Conclusión}

A pesar de que el mundo está en continua evolución, transformado por la globalización y la fragmentación, incluso la 'fractalization ${ }^{77}$, los principios y objetivos establecidos en el preámbulo y en el Artículo 1 de la carta de la ONU todavía son válidos. El multilateralismo o la gestión conjunta de los asuntos globales todavía son elementos pertinentes para las necesidades caleidoscópicas del presente, y dentro del plural, complejo e interdependiente mundo actual. Desde este punto de vista, las misiones y las acciones de la ONU deben convencer a los distintos actores del sistema internacional de que "el poder yace en la acción conjunta"78. Sin embargo, estos principios y objetivos también deben adaptarse al mundo actual mediante estructuras revitalizadas como un Consejo de Seguridad reformado y más representativo, una relación más estrecha entre la ONU y las organizaciones regionales y la emergencia de una sociedad civil internacional más fuerte y mejor organizada, que actúe como salvaguarda de las inmoderadas consecuencias de la globalización. La ONU también debe ser dotada de estructuras que ayuden al Consejo de Seguridad a decidir y actuar de forma anticipada y con métodos preventivos. Por lo tanto, se debería crear un centro de análisis y previsión, además de un centro de prevención de conflicto, ligado al Consejo de Seguridad. La ONU también debe tener en cuenta más a menudo los 'nuevos' valores internacionales: los derechos de los seres humanos, la ética moral, la democracia, un contrato social renovado ${ }^{79}$.

La Organización de Naciones Unidas es y sigue siendo el mejor instrumento para la diplomacia multilateral y un mecanismo esencial de 'gobierno global', construido para los estados y permanentemente a su disposición ${ }^{80}$. Sin embargo, una reforma positiva de la Organización se

${ }^{n}$ El concepto de 'fractalization' indica la multidimensionalidad, la fragmentación de mundo policéntrico actual. Véase articulo de Jcan de Mailiard, "Le crime à venir: vers une société fractale", Le débat, N 94, marzoabril de 1997.

"Mythes et réalités de la mondialisation", Esprit, noviembre de 1996.

${ }^{7}$ Véase Federico Mayor (con la colaboración de Jérôme Bindé), Un monde nouveau, (París, Éditions Odile Jacob, 1999), 526 pp. Véase también Federico Mayor y Jérôme Bindé, “The 21st Century: A Better World or a Brave New World", Foresight, 1(5):389-391, octubre de 1999.

${ }^{80}$ Un mecanismo que puede mejorarse mediante una mayor integración de numerosos y nuevos actores en los métodos de toma de decisiones de las Naciones Unidas, mediante un mayor grado de democracia en el sistema y mediante la resolución de las tensiones actuales entre los valores humanos e institucionales. Véasc A. J. R. Groom, "Globai Governance and the United Nations", en The United Nations at Fifty: Prospects and Retrospect, (Nueva Zelandia, 1996), p. 297. 
topa con dos problemas principales: la inversión desigual de los estados y la percepción del interés nacional. En un mundo global, el interés nacional ya no se detiene en los límites de un territorio. Los problemas nacionales son, cada vez más, también problemas internacionales, o al menos, tienen consecuencias mundiales. Por ejemplo, el control del agua no puede negociarse según el interés nacional, sino dentro de un marco regional. Los estados deberían reforzar la cooperación entre ellos y confiar más los unos en los otros. Por ahora, la conclusión del proceso de reforma de las Naciones Unidas (tanto el estructural como el conceptual) se topa con el deseo de cada estado miembro de maximizar sus intereses o su postura en el seno de cada grupo de trabajo, órgano, institución o programa.

Esta limitación debe ser superada a través de la adopción de una visión global común respecto a los problemas del mundo y mediante el reconocimiento de que la ONU no está, ni actúa, al mismo nivel que los estados. Sobre todo, la ONU no actúa contra ellos sino en beneficio de sus poblaciones. Este es el papel complementario de la ONU que debería destacarse. Puede, mejor que los estados, actuar como árbitro, como iniciador y como poder estabilizador y regulador dentro del sistema. Por lo tanto, es menos necesario cambiar la institución o modificar los textos (aunque sea necesaria la 'limpieza' de algunos artículos), que aplicar los artículos de la Carta de forma estricta. Los vínculos entre paz, desarrollo y seguridad humana ya están escritos entre líneas en la Carta.

Para concluir, la ONU debe recuperar su relevancia desde el punto de vista más general de los valores. Lejos de menospreciar los valores individuales (pero sólo aquellos que conciernen la intromisión y la intolerancia), las acciones de la ONU permiten que todos seamos conscientes de la necesidad de unos valores universales y comunes, cuyo único propósito es proteger el planeta y la herencia que han dejado varias generaciones y civilizaciones. Como dice Edgar Morin, "de la misma manera que debe establecerse una comunicación viva entre pasado, presente y futuro, también debe establecerse una comunicación viva y permanente entre las singularidades culturales, étnicas y nacionales y el universo concreto de una Madre Naturaleza para todos"81. Por lo tanto, "la adopción de una visión global de las cosas es la principal condición para nuestra supervivencia"82.

"Edgar Morin, "Our Common Home", The UNESCO Courier, noviembre de 1995.

${ }^{32}$ Federico Mayor, "The Price of Peacc", The UNESCO Courier, noviembre de 1995. 
Existen muchas maneras de dar más autoridad, credibilidad y eficacia a la Organización de Naciones Unidas, a su sistema y a sus acciones. Esto es imprescindible para mejorar las condiciones de vida de este mundo y la comprensión de los actores que evolucionan en la esfera internacional. A fin de compensar por la falta de reflexión y voluntad política (que a veces refleja la falta de buena voluntad de los estados) debería llevarse a cabo una reflexión profunda desde centros o estructuras dedicadas a la previsión, a la prevención de crisis y a la observación del funcionamiento de nuestras sociedades. Esta reflexión que trata la reforma de la ONU en realidad va más allá: también implica la redefinición de nuestra relación con el tiempo, hacer evolucionar o cambiar la manera en la que funciona la democracia (hacia una mayor participación de todos), la consciencia de la unidad de destino del planeta y recuperar el sentido de interés común. La ONU puede ser fuerte de nuevo si los actores internacionales consiguen alcanzar "el reto... actualizar el ideal sin perder el sentido de la realidad"83.

${ }^{83}$ David Caron, en Proceedings of the 87th Anmual Meeting of the American Society of International Law, (Washington D.C. 1993), p. 310. 